\title{
Total Hepatectomy Model in Pigs: Revised Method for Vascular Reconstruction Using a Rigid Vascular Prosthesis
}

\author{
M.N. Sosef T.M. van Gulik \\ Department of Surgery (Surgical Laboratory), Academic Medical Center, The University of Amsterdam, \\ Amsterdam, The Netherlands
}

\section{Key Words}

Animal model · Pigs · Hepatectomy $\cdot$ Hepatic failure • Bioartificial liver

\begin{abstract}
Total hepatectomy in animals provides an irreversible model of acute liver failure. Vascular reconstruction in this model of acute liver failure was modified and characterized for the use of assessment of liver support systems. Pigs underwent total hepatectomy and a rigid three-way transparent polyethylene vascular conduit was used to replace the retrohepatic caval vein and to shunt the portal venous blood to the caval vein. Placement of the vascular conduit in conjunction with excision of the liver was completed in 10-22 min without the need of a temporary veno-venous bypass. A survival study conducted in 5 animals showed a mean survival time of $46 \pm 6 \mathrm{~h}$. Baseline and $4 \mathrm{~h}$ postoperative hemoglobin levels were not different, and plasma ammonia levels rose to more than 30 -fold of baseline values. All animals died of cardiac arrhythmias and irreversible shock. Total hepatectomy in the pig using a three-way portal-venous conduit is a reliable and well-reproducible animal model of acute liver failure for evaluation of liver assist devices.
\end{abstract}

Copyright $(2004$ S. Karger AG, Basel

\section{Introduction}

In the development of a bioartificial liver (BAL), one of the essential steps to be taken before clinical application is assessment of its efficacy and safety in a large animal model of fulminant hepatic failure. A variety of animal models of hepatic failure have been elaborated in the past, based on liver devascularization, total hepatectomy, the administration of hepatotoxic drugs or a combination of surgical and intoxication models. However, none of these models is able to entirely mimic the pathophysiology of fulminant hepatic failure in humans and is sufficiently reproducible as well.

The total hepatectomy model renders an anhepatic animal. Advantages of the anhepatic model are its clarity, reproducibility and its potential to assess the biochemical capacity of a BAL device in vivo in the absence of toxic products leaking out of, or produced by the native liver. Obviously, the release of products of necrosis and signal transmitters, seen as an important factor in the pathophysiology of fulminant liver failure, is absent in the anhepatic model.

Application of the anhepatic model in pigs requires reconstruction of the caval vein in combination with drainage of the portal venous system to prevent splanchnic congestion. The intrahepatic part of the caval vein is either dissected off the liver in combination with a porto-

\begin{tabular}{ll}
\hline KARGER & ( ) 2004 S. Karger AG, Basel \\
0014-312X/04/0361-0008\$21.00/0 \\
$\begin{array}{l}\text { Fax +4161306 1234 } \\
\begin{array}{l}\text { E-Mail karger@karger.ch } \\
\text { www.karger.com }\end{array}\end{array}$ & $\begin{array}{l}\text { Accessible online at: } \\
\text { www.karger.com/esr }\end{array}$
\end{tabular}

Prof. Dr. T.M. van Gulik

Surgical Laboratory IWO 1-155

Academic Medical Center, Meibergdreef 9

NL-1105 AZ Amsterdam (The Netherlands)

Tel. +31 20 5665570, Fax +31206976621, E-Mail t.m.vangulik@amc.uva.nl 
caval shunt, or is excised in conjunction with the whole liver, followed by reconstruction of vascular portal-caval and caval-caval continuity. Usually, a Dacron prosthesis has been used as a vascular conduit $[1,2]$.

In an initial study, the anhepatic pig model as described by Mazziotti et al. [1] was used in our institution to evaluate a newly devised BAL (AMC-BAL). A total hepatectomy was performed after construction of a portocaval shunt. Caval continuity was restored with use of a Dacron prosthesis. In order to assess at least $24 \mathrm{~h}$ of treatment with the AMC-BAL, the animals remained anesthetized and mechanically ventilated until death. All 4 animals of this series showed severe coagulation disorders after 24-30 h, with continuous and diffuse bleeding through the vascular anastomoses and through the mesh of the Dacron prosthesis. This limitation of the animal model prompted us to develop a technique of vascular reconstruction using a rigid polyethylene three-way prosthesis. The concept is based on the Y-shaped Pyrex-glass cannula as used by Firor and Stinson [3] in 1929 with total hepatectomy in dogs.

The aim of this study was to assess the efficacy of a self-made, three-way prosthesis after total hepatectomy in pigs and at the same time, characterize this anhepatic model for prolonged assessment of liver assist devices.

\section{Methods}

Adult white female pigs were used, weighing between 37 and $71 \mathrm{~kg}$ (mean $51 \pm 9 \mathrm{~kg}$ ). All procedures were approved by the institutional guidelines of the Animal Ethical Committee of the University of Amsterdam.

\section{Anesthesia}

After fasting overnight, induction of anesthesia was achieved with intramuscular administration of ketamine $(10 \mathrm{mg} / \mathrm{kg}$; Nimatec $^{\circledR}$, Eurovet, Bladel, The Netherlands), azaperon ( $2 \mathrm{mg} / \mathrm{kg}$; Stresnil ${ }^{\circledR}$, Janssen Pharmaceutica, Tilburg, The Netherlands) and atropine $(0.02 \mathrm{mg} / \mathrm{kg})$. After inhalation of a mixture of $\mathrm{O}_{2}: \mathrm{NO}_{2}(2: 3)$ and isoflurane (0.4-1\%, Abbott Laboratories Ltd, Queensborough, Kent, UK), pigs were intubated with a cuffed endotracheal tube and ventilated on a mixture of $\mathrm{O}_{2}$ and air. Anesthesia was maintained by intravenous administration $(0.5 \mathrm{ml} / \mathrm{kg} / \mathrm{h}$, after total hepatectomy $0.2 \mathrm{ml} /$ $\mathrm{kg} / \mathrm{h})$ of a mixture of sufentanilcitrate $(20 \mathrm{mg} / \mathrm{l}$, Janssen-Cilag, Tilburg, The Netherlands) and ketamine $(20 \mathrm{~g} / \mathrm{l})$. Muscle relaxation was obtained by intravenous administration $(2 \mathrm{ml} / \mathrm{h})$ of pancuronium bromide ( $2 \mathrm{mg} / \mathrm{ml}$, Organon Teknika BV, Boxtel, The Netherlands). Arterial and venous lines were inserted in the right axillary artery and internal jugular vein for blood sampling and for continuous monitoring of arterial pressure and central venous pressure, respectively.

\section{Surgical Procedure}

A laparotomy was performed using an abdominal midline incision. A urine catheter was sutured in the bladder in order to monitor urine production. The liver was freed of all peritoneal attachments. The common bile duct and hepatic arteries were ligated and transected. The suprahepatic cava, suprarenal cava and portal vein were dissected free, with careful ligation of the remaining lymphatic tissue in the hepatoduodenal ligament. The vascular prosthesis used was $15 \mathrm{~cm}$ long, made of transparent polyethylene with an inner diameter of $12 \mathrm{~mm}$ (caval vein junction sites) and $10 \mathrm{~mm}$ (portal site). Parts were glued together, and a Luer Lock connection point in the portal part allowed temporary insertion of a $\mathrm{Ch} 6$ occlusion balloon in the caval part. The prosthesis was sterilized in glutaraldehyde solution, washed thoroughly and flushed with a heparin solution $(10 \mathrm{U} / \mathrm{ml}$, Leo BV, Weesp, The Netherlands) before use.

The portal vein was cross-clamped and cannulated (marking the start of the anhepatic state) after which the liver was flushed with $1,000 \mathrm{ml}$ of cold $\left(4^{\circ} \mathrm{C}\right)$ Ringers glucose solution (NPBI International BV, Emmer Compascuum, The Netherlands). After subsequent temporary clamping of the supra- and infrahepatic caval vein, the rigid three-way prosthesis was inserted upwards through a caval venotomy, passing the entry sites of the hepatic veins and fixed cranially and caudally of the liver with tourniquets around the caval vein. Caval blood flow was restored after desufflation, unclamping and retraction of the occlusion balloon in the portal part of the prosthesis. Portal blood flow was subsequently shunted to the systemic circulation by connecting the portal vein to the side-port of the prosthesis, after which the balloon could be removed (fig. 1). The liver was removed in toto after incising the circumferences of supra- and infrahepatic caval vein and in between these margins, the posterior wall of the caval vein. The transparency of the prosthesis allowed visual assessment of portocaval blood flow. No decompressive venous bypass was required. No blood transfusions were given during the procedure.

\section{Postoperative Care}

Postoperatively, all animals were kept under full anesthesia until death. Fluids were administered intravenously as clinically indicated (electrolyte solutions and Ringers lactate, NPBI International BV; eloHaes, Fresenius BV, 's-Hertogenbosch, The Netherlands). Phenylephrine $(10 \mathrm{mg} / \mathrm{ml} ; 2-25 \mathrm{ml} / \mathrm{h})$ was administered intravenously when necessary to maintain mean arterial blood pressure $>55 \mathrm{~mm} \mathrm{Hg}$. Animals were kept on volume-controlled, positive pressure ventilation during the experiment. Inspiratory peak pressure, capnography, direct arterial blood pressure, and central venous blood pressure were monitored continuously and corrected to physiological values. Body temperature was maintained at $38^{\circ} \mathrm{C}$ using a heated mattress. A $20 \%$ glucose solution was infused when plasma glucose levels fell below $8 \mathrm{mmol} / \mathrm{l}$. Every $12 \mathrm{~h}$, the animals received $1 \mathrm{~g}$ of ceftriaxon intravenously (Hoffmann-La Roche, Basel, Switzerland). Autopsy was ultimately performed in all animals.

\section{Laboratory Assessments}

Arterial blood samples were obtained preoperatively, at the moment of total hepatectomy (defined as $\mathrm{t}=0 \mathrm{~h}$ ) and at 4-hourly intervals post-hepatectomy until death, for determination of hemoglobin, leukocyte and thrombocyte count, albumin, lactate, creatinine, BUN, AST, ALT, ammonia and prothrombin time by standard laboratory techniques. Ammonia was measured by means of a spectophotometric method (ACA SX, Dupont). 


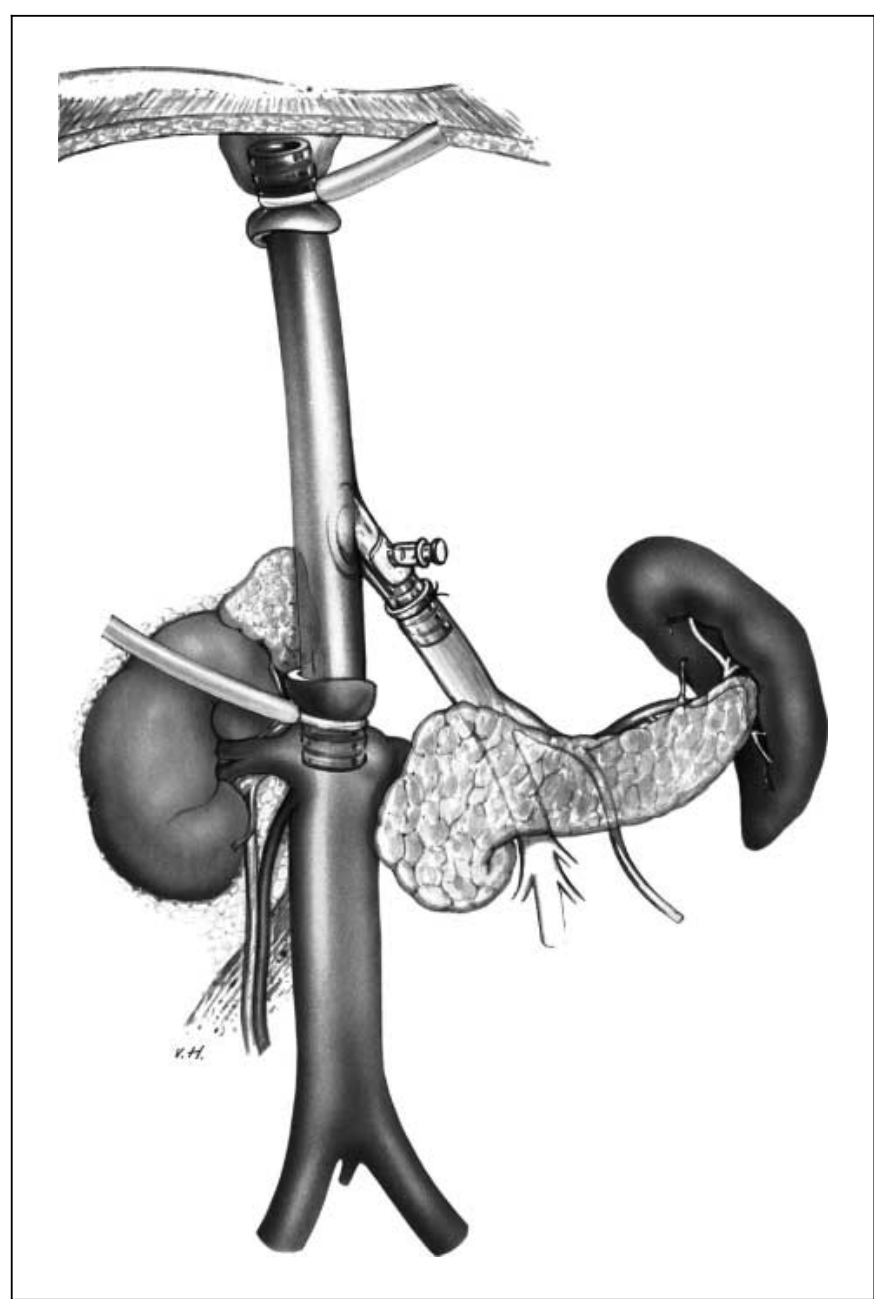

Fig. 1. Schematic drawing of the rigid prosthesis, serving as a vascular conduit between portal and caval veins after total hepatectomy.

\section{Results}

A total hepatectomy with use of the rigid prosthesis was performed in 20 animals. The surgical procedure proved to be relatively easy and straightforward, with a short learning curve of 2-3 animals. Time between clamping of the portal vein and complete restoration of portocaval blood flow was $16 \pm 4$ min (mean $\pm \mathrm{SD}$; range 10-22 min). Intraoperative blood loss was $270 \pm 225 \mathrm{ml}$, including electrolyte solution leaking out of the cava during excision of the liver. During clamping of the caval and portal veins progressive hypotension and tachycardia was observed, with mean arterial pressures of approximately $40 \mathrm{~mm} \mathrm{Hg}$ after 8-10 min. After restoration of flow, a good hemodynamic recovery was seen in all animals.

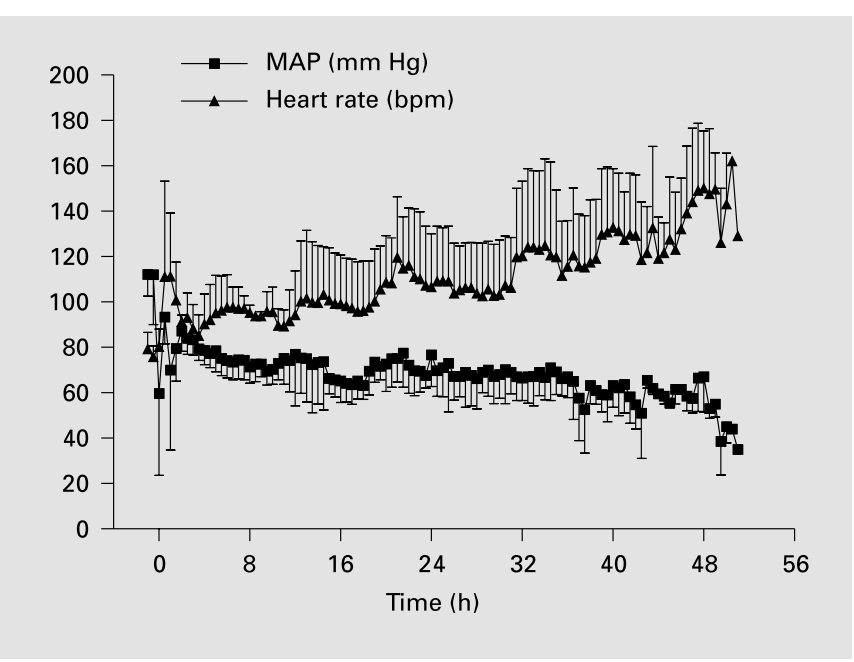

Fig. 2. Mean $( \pm \mathrm{SD})$ arterial pressure (lower line) and heart-rate (upper line) after total hepatectomy (at $\mathrm{t}=0 \mathrm{~h}$ ). Phenylephrine was used when clinically indicated.

Bowels regained their normal color within 2 min after unclamping the portal vein.

Technical problems led to the termination of the experiments in 3 animals: one caval lesion during dissection prior to placement of the prosthesis, one portal vein laceration and one gastric congestion and bleeding after tying the splenic vein (learning curve). Two experiments were terminated because of infectious complications: one pneumonia, and one Pseudomonas spp. septicemia. Five anhepatic animals received postoperative supportive treatment until death including positive pressure ventilation, and showed a mean survival time of $46 \pm 6 \mathrm{~h}$ (37, $41,50,50$ and $51 \mathrm{~h}$ ). In order to control hypotension, phenylephrine was administered starting 20-24 h after hepatectomy, resulting in a progressive tachycardia (fig. 2). Eventually, all animals died due to irreversible shock or cardiac arrhythmias.

Hemoglobin levels $4 \mathrm{~h}$ post-hepatectomy did not significantly differ from baseline values $(5.0$ vs. $5.1 \mathrm{mmol} / 1$ respectively). Baseline and postoperative laboratory values are reported in table 1. Hemoglobin, thrombocyte count and albumin show a gradual decrease to $35-45 \%$ of baseline values just before death. Blood ammonia levels gradually rose more than 30 -fold, while lactate levels only increased a few hours before death when the animals became hemodynamically unstable and required a high dose of inotropic vasopressor agents.

At autopsy, no signs of portal congestion were noticed. $100-350 \mathrm{ml}$ of serosanguinolent fluid was found in the 
Table 1. Baseline laboratory blood values compared with values at different time points $(\mathrm{h})$ before death $\left(\mathrm{T}_{\mathrm{d}}\right)$. No blood transfusions or albumin supplementations were given

\begin{tabular}{|c|c|c|c|c|c|c|}
\hline & Baseline & $\mathrm{T}_{\mathrm{d}}-36$ & $\mathrm{~T}_{\mathrm{d}}-24$ & $\mathrm{~T}_{\mathrm{d}}-12$ & $\mathrm{~T}_{\mathrm{d}}-4$ & $\mathrm{~T}_{\mathrm{d}}$ \\
\hline Hemoglobin, mmol/l & $5.14 \pm 0.51$ & $4.76 \pm 1.23$ & $4.46 \pm 0.96$ & $3.93 \pm 1.23$ & $2.78 \pm 1.15$ & $2.33 \pm 0.87$ \\
\hline Leukocytes, $10^{9} / 1$ & $12 \pm 2.4$ & $28 \pm 4.1$ & $33 \pm 11.6$ & $28 \pm 12.7$ & $27 \pm 9.7$ & $23 \pm 10.8$ \\
\hline Thrombocytes, $10^{12} / 1$ & $267 \pm 68$ & $216 \pm 64$ & $161 \pm 54$ & $131 \pm 58$ & $106 \pm 19$ & $95 \pm 13$ \\
\hline Albumin, $\mathrm{g} / 1$ & $28 \pm 2.9$ & $17 \pm 2.9$ & $15 \pm 4.0$ & $15 \pm 3.8$ & $12 \pm 4.2$ & $10 \pm 3.3$ \\
\hline Lactate, $\mathrm{mmol} / \mathrm{l}$ & $1.10 \pm 0.47$ & $1.54 \pm 0.58$ & $1.75 \pm 0.47$ & $1.95 \pm 0.66$ & $2.55 \pm 0.72$ & $5.63 \pm 3.71$ \\
\hline Creatinine, $\mu \mathrm{g} / 1$ & $82 \pm 17$ & $95 \pm 17$ & $80 \pm 15$ & $121 \pm 38$ & $153 \pm 58$ & $186 \pm 67$ \\
\hline BUN & $2.84 \pm 1.30$ & $1.22 \pm 0.82$ & $1.08 \pm 0.55$ & $1.25 \pm 0.53$ & $1.50 \pm 0.75$ & $1.70 \pm 0.59$ \\
\hline AST, U/1 & $25 \pm 12$ & $198 \pm 74$ & $220 \pm 5$ & $280 \pm 109$ & $253 \pm 86$ & $258 \pm 81$ \\
\hline ALT, U/1 & $38 \pm 12$ & $36 \pm 12$ & $35 \pm 14$ & $34 \pm 12$ & $27 \pm 10$ & $26 \pm 9$ \\
\hline Ammonia, mol/1 & $57 \pm 32$ & $372 \pm 94$ & $322 \pm 96$ & $636 \pm 429$ & $1,347 \pm 888$ & $1,843 \pm 1,215$ \\
\hline PT, s & $11 \pm 0.4$ & $19 \pm 4.4$ & $>40$ & $>40$ & $>40$ & $>40$ \\
\hline
\end{tabular}

abdominal cavity of all animals. All grafts were patent without signs of thrombosis and there were no signs of bleeding from the connection sites between prosthesis and veins. Brain autopsy was not performed.

The remaining 10 animals were connected to the AMC-BAL for evaluation of its efficacy in the anhepatic pig as has been reported elsewhere [4].

\section{Discussion}

This study describes a modified surgical technique of performing a total hepatectomy in pigs, to serve as an animal model of acute liver failure that is suitable for testing of temporary liver support treatments. For a limited number of animals, the postoperative course until death is reported in detail.

Unfortunately, there is no animal model of acute liver failure that is pathophysiologically and metabolically identical to fulminant hepatic failure in humans, and is at the same time well reproducible and potentially reversible. Specific study objectives and questions will ultimately lead to the choice of a suitable animal model. The most frequently employed models use high doses of hepatotoxins such as galactosamine and acetaminophen, or are based on surgical anhepatic or devascularization procedures.

Drug-toxicity models probably provide the most clinically relevant models for studies on the disease itself. An important disadvantage which is especially relevant in controlled treatment studies, is the difficulty of reproducibility and extrahepatic hepatotoxicity [5]. Surgical mod- els have less pathophysiologic similarities with fulminant hepatic failure than toxic models, but are easier to reproduce and, in case of total hepatectomy, offer a way to measure absolute function of a BAL device in vivo. They can be separated into those that provide functional hepatectomy, leaving ischemic liver in situ deprived of its afferent blood supply, or techniques that involve anatomic excision of the liver. Functional hepatectomy involves creating a terminal portal-caval shunt and (in a one-stage procedure or in separate steps) skeletonizing the whole liver with ligation of the hepatic artery and all other, collateral arterial branches. This complete liver ischemia model resembles clinical fulminant failure to the extent that the failing liver remains in situ and is allowed to release products of necrosis in the systemic circulation, which allegedly are responsible for the toxic syndrome of acute liver failure. Survival times of up to $33.1 \pm 3 \mathrm{~h}$ (mean \pm SEM) have been shown in pigs with complete liver ischemia [6], as compared to survival times of up to $27 \mathrm{~h}$ in pigs rendered anhepatic [2].

Mann and Magath [7], in 1921, were the first to perform a total hepatectomy in dogs, and were followed by other groups reporting on total hepatectomies in rats $[8,9]$ and pigs $[2,10,11]$. The main objectives of the earlier studies were to study metabolism of the liver and the pathophysiology of the anhepatic state. Development and refinement of treatment regimens of hepatic failure were a second objective, now experiencing renewed interest with the recent development of BALs. The pig is the most suitable, large animal to evaluate liver support systems because of, among other reasons, its close anatomical, physical and biochemical resemblance to humans. The use of a 
knitted, Dacron prosthesis for vascular reconstruction after total hepatectomy in pigs led to severe bleeding complications after $24-30 \mathrm{~h}$ of anhepatic state, imposing significant limitations to the model and therefore was abandoned in this study.

The procedure described in this study proves to be straightforward and safe. The limited variation in survival times and the continued, postoperative supportive care offer a therapeutic window suitable for assessment of treatment with a BAL. The hepatectomy involved a relatively minor surgical trauma as demonstrated by the rapid stabilization of the animal after surgery. Blood transfusions were not necessary owing to minimal intraoperative blood loss and flushing of the liver prior to clamping of the caval veins. The significant decrease in albumin levels can be attributed to lacking production of albumin as well as intra-abdominal leakage of lymphatic fluid, despite accurate ligation of the lymphatic vessels in the hepatoduodenal ligament and the peritoneal adhesions. Assessment of ICP was attempted in the initial series but was relinquished because of severe bleeding problems at the craniotomy site with prolonged duration of the anhepatic state.

Terblanche and Hickman [12] stated six criteria for a satisfactory animal model of fulminant hepatic failure:
(1) reversibility (animals should be able to survive if a suitable treatment were to be utilized), (2) reproducibility, (3) death from liver failure, (4) adequate therapeutic window (large enough to assess effects of treatment), (5) large animal type of model, and (6) minimal hazard to personnel. Clearly, the first criterion cannot be matched in the anhepatic model. Our data show that the employed technique of total hepatectomy in pigs meets the other five criteria. Advantages of our prosthesis are the simple procedure for insertion combined with minimal blood loss, and its transparency in order that blood flow can be checked after placement. Furthermore, there is no need for temporary venous decompression owing to short clamping times. The tight tourniquets that fix the prosthesis to the veins abolish bleeding complications even in situations after prolonged anhepatic state when blood coagulation is close to absent.

In conclusion, application of a three-way polyethylene vascular prosthesis in conjunction with total hepatectomy provides a reliable, safe and effective animal model of acute liver failure allowing the evaluation of liver assist devices for an extended period of time. Longer survival times have been achieved with this prosthesis, when compared with the knitted, Dacron prosthesis.

\section{References}

1 Mazziotti A, Bernardi M, Antonini L, Dioguardi FS, Bellusci R, Papa V, Tacconi C, Gasbarrini G, Cavallari A, Possati L: Plasma amino acid patterns in experimental acute hepatic failure: Comparison between hepatectomy and liver devascularization in pigs. Surgery 1981; 90:527-534.

2 Filipponi F, Boggi U, Meacci L, Burchielli S, Vistoli F, Bellini R, Prota C, Colizzi L, Kusmic C, Campani D, Gneri C, Trivella MG, Mosca F: A new technique for total hepatectomy in the pig for testing liver support devices. Surgery 1999; 125:448-455.

3 Firor WM, Stinson E: Total extirpation of the dog's liver in one stage. Bull J Hopkins Hosp 1929;44:138-148.

4 Sosef MN, Abrahamse LS, van De Kerkhove MP, Hartman R, Chamuleau RA, van Gulik TM: Assessment of the AMC-bioartificial liver in the anhepatic pig. Transplantation 2002;73: 204-209.
5 Newsome PN, Plevris JN, Nelson LJ, Hayes PC: Animal models of fulminant hepatic failure: A critical evaluation. Liver Transpl 2000; 6:21-31.

6 Flendrig LM, Calise F, Di Florio E, Mancini A, Ceriello A, Santaniello W, Mezza E, Sicoli F, Belleza G, Bracco A, Cozzolino S, Scala D, Mazzone M, Fattore M, Gonzales E, Chamuleau RA: Significantly improved survival time in pigs with complete liver ischemia treated with a novel bioartificial liver. Int J Artif Organs 1999;22:701-709.

7 Mann FC, Magath TB: The effect of total removal of the liver. Trans Sect Pathol Physiol Am Med Assoc 1921, pp 419-511.

8 Olafsson S, Gottstein J, Blei AT: Brain edema and intracranial hypertension in rats after total hepatectomy. Gastroenterology 1995;108: 1097-1103.
9 Engelbrecht GH, Hickman R, Kahn D: Onestage total hepatectomy in the rat using microvascular anastomoses. Microsurgery 1999;19: 95-97.

10 Tonnesen K, Balling H: Cerebral flow and metabolism in experimental liver failure. A comparison between hepatectomy and total hepatic devascularization in pigs. Acta Chir Scand 1986;152:439-445.

11 Vistoli F, Boggi U, Bellini R, Colizzi L, Kusmic C, Burchielli S, Campani D, Gneri C, Trivella MG, Filipponi F, Mosca F: A standardized pig model of total hepatectomy for testing liver support systems. Transplant Proc 2000;32: 2723-2725.

12 Terblanche J, Hickman R: Animal models of fulminant hepatic failure. Dig Dis Sci 1991;36: 770-774. 